\title{
Organizaciones innovadoras a partir de la interacción con la universidad: casos exitosos
}

\author{
MARÍA EUGENIA MORALES RUBIANO, MSc. ${ }^{1,2,3}$ \\ Docente investigadora, Universidad Militar Nueva Granada, Colombia. \\ maria.morales@unimilitar.edu.co \\ KATHERINE PINEDA MÁRQUEZ \\ Joven investigadora, Universidad Militar Nueva Granada, Colombia. \\ kpinedamar@gmail.com \\ KAROLINA ÁVILA MARTÍNEZ \\ Universidad Militar Nueva Granada. Colombia. \\ karo_9122@hotmail.com
}

\section{RESUMEN}

Se busca mostrar estrategias y casos exitosos de colaboración entre universidades y organizaciones del entorno que han generado innovación a partir de su interacción. Para esto, se revisó literatura en bases de datos científicas que muestran innovaciones colaborativas en países de la región y en otros países. También se obtuvo información en páginas web de diez universidades destacadas en el Ranking Web de Universidades del Mundo 2011. Los resultados muestran que se han establecido actividades de cooperación entre universidades y otros actores a través de investigación colaborativa, desarrollo tecnológico y emprendimiento. Lo anterior evidencia retos para Latinoamérica y Colombia en el direccionamiento de actividades conjuntas para la innovación.

Palabras clave. Transferencia de conocimiento; interacción universidad entorno; innovación.

Recibido: 30-jun-11, corregido: 27-abr-12 y aceptado: 26-jun-12

$$
\text { Clasificación JEL: } \mathrm{O}_{31} ; \mathrm{O}_{32} ; \mathrm{O}_{33}
$$

@

\footnotetext{
1 Este documento fue seleccionado en la convocatoria para enviar artículos, Call for Papers, realizada en el marco del “II Simposio Iberoamericano de Estudios Gerenciales: Una mirada interdisciplinar a la innovación”, organizado por la revista académica Estudios Gerenciales bajo la dirección de la Facultad de Ciencias Administrativas y Económicas de la Universidad Icesi; el evento tuvo lugar los días 12, 13 y 14 de octubre de 2011, en la ciudad de Cali (Colombia). Este documento fue presentado en las sesiones simultáneas del área de "Innovación en relaciones organizacionales". 2 Artículo de investigación producto del desarrollo del proyecto ECO 919 "Estrategias y experiencias de la vinculación Universidad, Empresa y Estado en la Universidad Nacional de Colombia como referente competitivo para la Universidad Militar Nueva Granada- UMNG" y del proyecto Institucional Integración Universidad, Empresa y Estado, financiados y ejecutados por la Universidad Militar Nueva Granada.

3 Autor para correspondencia. Dirigir a: Carrera 11 No. 101-80 Bloque C, Bogotá, Colombia.
} 


\section{Innovative organizations based on the interaction with universities: successful cases}

The purpose of this article is to present successful strategies and cases of collaboration between local universities and organizations that have led to innovation on different levels thanks to their interaction. To this end, a literature review was conducted of scientific databases that discuss cases of collaborative innovations in countries in the region and other countries, and of information from the websites of ten leading universities according to the worldwide web ranking of universities in 2011. The results show that universities and other stakeholders have engaged in cooperation activities through collaborative research, technological development, and entrepreneurship efforts. This evidently poses challenges for Colombia and other Latin American countries in terms of guiding joint activities that foster innovation.

Keywords. Knowledge transfer; interaction; innovation.

\section{es}

\section{Organizações inovadoras a partir da interação com a universidade: histórias de sucesso}

O objetivo é mostrar estratégias e histórias de sucesso de colaboração entre universidades e organizações ambientais que geraram inovação a partir de sua interação. Para isso, foi realizada uma revisão da literatura em bases de dados científicos que mostram a inovação colaborativa em países da região e de outros países, e também obteve informações em sites de 10 universidades de ponta no ranking web das universidades do mundo de 2011. Os resultados mostram que estabeleceram atividades de cooperação entre as universidades e outras partes interessadas, através de pesquisa colaborativa, desenvolvimento tecnológico e empreendedorismo. A exposição anterior evidência os desafios para a América Latina e Colômbia para enfrentar ações conjuntas para a inovação.

Palavras-chave. Transferência de conhecimentos; interação universidade ambiente; inovação. 


\section{Introducción}

En el contexto actual, el conocimiento es el factor estratégico para la generación de ventajas competitivas e innovación en las organizaciones, razón por la cual la universidad, como entidad generadora de conocimiento, toma un rol protagónico, especialmente alrededor de la función de extensión o proyección social, que es la llamada a llevar al entorno los desarrollos que se hacen en docencia e investigación (Morales, Mira \& Arias, 2010).

De acuerdo con lo anterior, es evidente la tendencia a establecer mecanismos que permitan la interacción de las universidades con su entorno. En los países desarrollados, son frecuentes las innovaciones generadas en los parques tecnológicos, donde empresas, universidades y otras organizaciones trabajan colaborativamente (Casallas, Plata \& Pineda, 2011).

En América Latina el desarrollo de los parques tecnológicos es incipiente, aunque otros mecanismos permiten fomentar la innovación a partir de la interacción de diversos actores, como es el caso de la investigación colaborativa entre universidades y empresas, las iniciativas de emprendimiento universitario y el fortalecimiento de vínculos entre actores a partir de las pasantías o prácticas estudiantiles.

El objetivo de este trabajo es mostrar estrategias y casos exitosos de colaboración entre universidades y organizaciones del entorno que han generado innovación en diferentes niveles a partir de su interacción. Para dar respuesta a lo anterior el documento se estructura en cuatro partes: nociones importantes sobre interacción universidad-entorno, metodología usada para esta investigación, resultados y conclusiones.

\section{Nociones importantes sobre interac- ción universidad-entorno}

\section{La relación universidad y entorno}

En los últimos años y derivado de la importancia que tiene el conocimiento en la sociedad actual como fuente de riqueza, ha cobrado importancia la función de extensión universitaria, también llamada tercera misión de las universidades, la cual busca relacionar la universidad con su entorno, es decir, con la sociedad, las empresas y el Estado, lo anterior con el propósito de establecer acciones colaborativas que permitan responder a las necesidades reales de estos actores. En este sentido, estas interacciones han sido objeto de una gran cantidad de investigaciones que evidencian una relación positiva entre las vinculaciones universidadentorno y la intensidad con que se realizan actividades de investigación, desarrollo e innovación - I+D+I (Etzkowitz \& Leydesdorff, 2000; Pineda, Morales \& Ortiz, 2011, Sábato \& Botana, 1986).

\section{Estrategias y mecanismos de interacción}

Las estrategias y mecanismos de interacción varían según el contexto de los países (Torres, Dutrenit, Becerra \& Sampedro, 2009). En el caso de los países industrializados, existe una tendencia creciente a desarrollar alianzas estratégicas entre empresas y otras organizaciones con universidades, y a generar leyes y normatividades que sirvan de marco para poder realizar estas actividades.

Las principales estrategias y mecanismos de vinculación universidad-entorno son las spin offs, spin outs y start ups, ${ }^{4}$ las oficinas

4 Las start ups son los emprendimientos de base científica y tecnológica jóvenes, surgidos de procesos de incubación; las spin off son iniciativas empresariales de base científica y tecnológica que se encuentran en una fase madura y que pueden surgir desde las universidades a partir de procesos de investigación, pero que no modifican ni tienen impacto en la estrategia de la organización de origen. Finalmente, las spin outs son iniciativas empresariales que pueden 
de transferencia de tecnología (otris), 5 las incubadoras de empresas, los parques científicos y tecnológicos, ${ }^{6}$ las pasantías y prácticas profesionales, las consultorías, los programas de educación continuada, los centros de investigación cooperativa y de tecnología, las organizaciones regionales de innovación, las alianzas estratégicas, los centros o unidades empresariales y las políticas institucionales y nacionales, entre otros (Pineda et al., 2011).

No obstante, en los países en desarrollo, como es el caso de la región de Latinoamérica y otros países en regiones emergentes, los aspectos culturales y normativos en las organizaciones, la desarticulación entre la visión de las empresas, el Estado y las universidades, las divergencias en la forma de responder a las necesidades sociales de las regiones y la carencia de una masa crítica en I+D, además del desconocimiento $\mathrm{y}$ temor frente a aspectos de propiedad intelectual, han restringido los avances en las innovaciones que puedan darse a partir de las interacciones entre distintos actores.

\section{Metodología}

A partir de indagaciones previas sobre vínculos entre la universidad y su entorno (Pineda et al., 2011; Sutz, 200o), se identi-

surgir desde las universidades y que tienen por finalidad la independencia de algunos de los departamentos o divisiones de la organización.

5 Son organizaciones híbridas que se centran en identificar las necesidades tecnológicas de los sectores socioeconómicos y favorecer la transferencia de tecnología entre el sector público y el privado, contribuyendo así a la aplicación y comercialización de los resultados de la $\mathrm{I}+\mathrm{D}+\mathrm{I}$ generada en las universidades y centros públicos de investigación.

6 Los parques científicos y tecnológicos son estructuras que estimulan y gestionan el flujo de conocimiento y la tecnología entre universidades, instituciones de investigación, empresas y mercados; impulsan la creación y el crecimiento de empresas innovadoras mediante mecanismos de incubación y proporcionan otros servicios de valor añadido, así como espacios e instalaciones de gran calidad. ficaron casos específicos de innovaciones derivadas de estas interacciones en diversas organizaciones del mundo; cuyo análisis dejó ver algunas dinámicas que han permitido generar innovación en dichas organizaciones a partir del apoyo de universidades, como un ejemplo significativo para el caso colombiano.

La identificación de estos casos es producto de una revisión de literatura, principalmente en bases de datos científicas en inglés, español y portugués, que da cuenta de estudios realizados en países de la región y en otras regiones del mundo. Así mismo, esta revisión se fundamenta en la información obtenida en las páginas web de diez universidades destacadas en el Ranking Web de Universidades del Mundo (CSIC, 2011), tomando dos por cada región: Europa, Asia, Norteamérica, Latinoamérica y dos casos para Colombia.

De este modo, la muestra incluye: la Universidad de Cambridge y la Universidad de Oxford, en Europa; la Universidad Nacional de Taiwán y la Universidad de Tokio, en Asia; el Instituto Tecnológico de Massachusetts y la Universidad de Harvard, en Norteamérica; la Universidad de São Paulo y la Universidad Nacional Autónoma de México, en Latinoamérica; la Universidad Nacional de Colombia y la Universidad de Antioquia, en Colombia.

A partir de esta indagación, se identificó en cada una de las universidades estudiadas, un caso exitoso de vinculación con el entorno que representó una innovación para los actores involucrados, en productos, procesos, mercadotecnia u organizacional (OCDE \& EUROSTAT, 2005). Esto permitió realizar un análisis del contexto en cada caso e identificar oportunidades y recomendaciones para la innovación a partir de la interacción universidad-entorno, en Latinoamérica y especialmente en Colombia. 


\section{Resultados}

La revisión de literatura muestra que las actividades que han generado innovación en organizaciones asociadas son el emprendimiento de base científica y tecnológica, la investigación cooperada y las innovaciones tecnológicas (Sutz, 2000). Esto concuerda con los avances en las últimas décadas, tanto de universidades como de otras organizaciones, en sus procesos de investigación aplicada, propiedad intelectual y manejo de patentes; todos estos aspectos claves para que dicha interacción se lleve a cabo de forma efectiva.

Por regiones, se encuentra para el caso de Europa y Asia, que algunas empresas de estas regiones prefieren realizar alianzas con universidades estadounidenses que con las de su misma región, esto se debe a la agilidad de las universidad estadounidenses en la transferencia de conocimiento y tecnología (Hughes, 2006).

No obstante, en los últimos años se destaca el caso asiático, por la rápida adopción de las universidades de su rol en la innovación, que les ha permitido responder a las necesidades de las empresas, quienes son finalmente las principales demandantes de investigación en esta región del mundo (Hershberg, Nabeshima \& Yusuf, 2007).

En Latinoamérica, por su parte, se ha encontrado aversión al cambio por parte de todos los actores de la innovación, como son las empresas y otras organizaciones, las universidades y los gobiernos (De Paula \& Silveira, 2009), además de cierta desconfianza de las empresas respecto al aporte que pueden hacer las universidades y otras instituciones de ciencia y tecnología a la innovación. Sin embargo, se observa que estos aspectos han venido cambiando en los últimos años. Adicionalmente, la región se ha caracterizado por una limitada actividad investigativa y deficiencias en la infraes- tructura tecnológica y en la demanda de esta, teniendo en cuenta que las economías de la región siguen soportadas en su gran mayoría en actividades de tipo tradicional, con insuficientes avances en tecnología y procesos; lo cual también se ve reflejado en el caso de Colombia (Sutz, 200o).

Lo anterior contrasta con lo que sucede en el contexto norteamericano, donde especialmente en Estados Unidos, en los Estados de Massachusetts y California, existe un engranaje de oferta y demanda de servicios tecnológicos y transferencia de conocimiento entre universidades y empresas (Pertuzé, Calder, Greitzer \& Lucas, 2010; Shane, 2002).

En concordancia con todo lo anterior, se evidencian desarticulaciones entre el sector productivo y el académico en las regiones de Latinoamérica, Asia y aún en Europa; debido a limitaciones en las capacidades de transferencia de conocimiento, a dificultades en la interacción y a discusiones ideológicas frente al involucramiento de las universidades en la generación de innovaciones direccionadas al mercado.

\subsection{Revisión de casos en universidades des- tacadas por región}

Tomando como parámetro el estudio de algunos casos de innovaciones colaborativas en las dos principales universidades de cada región estudiada: Norteamérica, Europa, Asia, Latinoamérica y Colombia; se pudo evidenciar que estas universidades están colaborando con diversas organizaciones del entorno, transfiriendo conocimiento, tecnología e innovación.

Los casos aquí presentados de Europa (ver Tabla 1) nacieron como spin offs universitarias, desarrolladas desde la investigación al interior de la academia, que actualmente son empresas destacadas en el mercado, en sus respectivos sectores. En estos casos, se observa la incidencia de programas univer- 
Tabla 1. Innovaciones colaborativas de las principales universidades de Europa

\begin{tabular}{|c|c|}
\hline Universidad & Caso de innovación colaborativa \\
\hline $\begin{array}{l}\text { University of } \\
\text { Cambridge }\end{array}$ & $\begin{array}{l}\text { Enecsys (2011) es una empresa líder en la comercialización de micro conversores so- } \\
\text { lares de energía para usos residenciales y comerciales. Surgió como una spin out del } \\
\text { Departamento de Ingeniería de la Universidad de Cambridge. Esta compañía de tec- } \\
\text { nología limpia, recibió financiación a través del Programa Cambridge Enterprise, gra- } \\
\text { cias a su participación en el Cambridge Business Plan Competition. Tiene tecnología } \\
\text { patentada que ofrece mayor rendimiento e instalación de los micro-conversores } \\
\text { (University of Cambridge, 2011). La Universidad de Cambridge realiza competiciones } \\
\text { internas entre las ideas de negocio de los estudiantes, que han llegado a ganar patentes } \\
\text { o alianzas con empresas como Enecsys, Vortix, Angio Splice, Pharma Wheels, Auto TB, } \\
\text { Foresight Pharmaceuticals, entre otras (University of Cambridge, 2011). }\end{array}$ \\
\hline $\begin{array}{l}\text { University of } \\
\text { Oxford }\end{array}$ & $\begin{array}{l}\text { Oxitec es una empresa pionera en el control de insectos transmisores de enfermedades } \\
\text { y daños a cultivos (Oxitec, 2011). Esta empresa nació como una spin out de la Uni- } \\
\text { versidad de Oxford, siendo un claro ejemplo de emprendimiento desde la universidad } \\
\text { (University of Oxford, 2011). Esta iniciativa surgió cuando el genetista molecular, Dr. } \\
\text { Lucas Alphey, se dio cuenta que podía usar las técnicas aprendidas en sus estudios } \\
\text { fundamentales en moscas Drosophila para el control de insectos plaga, mediante el uso } \\
\text { de la genética para producir insectos estériles. La prevención de la reproducción por la } \\
\text { liberación masiva de insectos estériles reduce el número de insectos sin la necesidad de } \\
\text { insecticidas (University of Oxford, 2011). }\end{array}$ \\
\hline
\end{tabular}

Fuente: Elaboración propia.

sitarios de emprendimiento que vinculan a los estudiantes con el sector productivo a través de ideas atractivas para el sector privado y que aportan soluciones a necesidades sociales.

En la región de Asia se muestran casos exitosos de investigación y desarrollos conjuntos con organizaciones para obtener soluciones innovadoras en temas de ingeniería aplicada (ver Tabla 2).

Cabe resaltar que aunque las universidades asiáticas han logrado responder rápidamente a los cambios en la demanda de soluciones prácticas a problemas sociales y empresariales, disminuyendo la tendencia de las empresas asiáticas a buscar alianzas con universidades estadounidenses, aún cuentan con limitaciones burocráticas e ideológicas respecto a las actividades de interacción con el entorno para la innovación (Hershberg et al., 2007).

Por otro lado, las universidades líderes en Norteamérica (ver Tabla 3) muestran diver- sas estrategias y mecanismos para interactuar con el entorno, especialmente relacionados con la generación de start ups y de alianzas estratégicas. A partir del reconocimiento que han construido, estas universidades se han constituido en centros atractivos para las empresas y demás organizaciones de la sociedad que buscan soluciones efectivas.

En América Latina, las universidades líderes (ver Tabla 4) también muestran diferentes estrategias y mecanismos para interactuar con el entorno, especialmente a partir de las actividades de extensión. Sin embargo, son menos visibles mecanismos como spin offs, alianzas estratégicas o actividades consolidadas de emprendimiento universitario.

En América Latina, los casos referidos evidencian avances en investigación aplicada y colaborativa entre empresas consolidadas y centros de investigación universitarios y en el desarrollo de incubadoras de empresas universitarias. No obstante, la creación de empresas de base científica y tecnológica 
Tabla 2. Innovaciones colaborativas de las principales universidades de Asia

\begin{tabular}{|c|c|}
\hline Universidad & Casos de innovación colaborativa \\
\hline $\begin{array}{l}\text { National } \\
\text { Taiwan } \\
\text { University - } \\
\text { NTU }\end{array}$ & $\begin{array}{l}\text { Intel-NTU Connected Context Computing Center (Intel-NTU Center) es un centro de } \\
\text { investigación que explora la tecnología de comunicación máquina a máquina (M2M), } \\
\text { también llamada IOT "Internet Of Things", con la cual las máquinas pueden ser ligadas } \\
\text { internamente para intercambiar información y tomar decisiones independientemente } \\
\text { (Intel-NTU, 2011). Este es un caso de investigación colaborativa entre la importante } \\
\text { compañía proveedora de tecnologías de computación Intel y la NTU. Este es uno de } \\
\text { los proyectos más importantes y promisorios para las próximas décadas, que aportará } \\
\text { a temas como la eficiencia de energía, reducción de emisiones de carbono, transporte } \\
\text { inteligente, cuidado de la salud, suministro de alimentos, prevención de desastres y de } \\
\text { terrorismo y espacio de vida inteligente. Dicho centro de investigación está establecido } \\
\text { en el campus de la NTU (NTU, 2011). }\end{array}$ \\
\hline $\begin{array}{l}\text { University } \\
\text { of Tokyo }\end{array}$ & $\begin{array}{l}\text { тото es una empresa manufacturera de servicios sanitarios que realizó investigaciones } \\
\text { colaborativas con el laboratorio Fujishima-Hashimoto de la Universidad de Tokio con } \\
\text { el propósito de identificar técnicas basadas en las propiedades fotocatálicas del dióxido } \\
\text { de titanio, con múltiples aplicaciones anti-suciedad, anti-bacterias y anti-empañe. Estas } \\
\text { investigaciones se tradujeron en patentes conjuntas, artículos publicados en journals de } \\
\text { amplia difusión y novedosos productos como pisos y vidrios anti-bacteriales y auto-lim- } \\
\text { piables, además de baños que descomponen bioquímicamente los compuestos orgánicos } \\
\text { (Baba, Yarime \& Shichijo, 2010; Shahid \& Nabeshima|, 2007). Al respecto, La división de } \\
\text { relaciones universidad-empresa de la Universidad de Tokio señala que las investigaciones } \\
\text { adquieren valor cuando hacen contribuciones efectivas a la sociedad (University of To- } \\
\text { kyo, 2010). }\end{array}$ \\
\hline
\end{tabular}

Fuente: Elaboración propia.

Tabla 3. Innovaciones colaborativas de las principales universidades de Norteamérica

\begin{tabular}{|c|c|}
\hline Universidad & Casos exitosos de innovaciones colaborativas \\
\hline $\begin{array}{l}\text { Massachu- } \\
\text { setts Institute } \\
\text { of Technol- } \\
\text { ogy - MIT } \\
(2011)\end{array}$ & $\begin{array}{l}\text { T2Biosystems es un spin off originada al interior del MIT por investigadores en las áreas } \\
\text { de ingeniería, medicina y biología. Esta empresa ha creado sistemas de rápido diag- } \\
\text { nóstico y tratamiento de enfermedades, proveyendo medición cuantitativa y cualitativa } \\
\text { en el análisis de muestras de sangre a través de una tecnología que combina resonancia } \\
\text { magnética, nano-partículas y pruebas moleculares, aportando beneficios como la real- } \\
\text { ización de pruebas rápidas para patógenos que actualmente requieren días para arrojar } \\
\text { resultados, como en el caso de los hongos y bacterias. Esto significa una disminución } \\
\text { de riesgos en los pacientes por la demora de resultados de diagnóstico, y por ende, en } \\
\text { su tratamiento (T2Biosystems, 2011). }\end{array}$ \\
\hline $\begin{array}{l}\text { Harvard } \\
\text { University }\end{array}$ & $\begin{array}{l}\text { Nano-Terra Inc. es una start-up fundada por George M. Whitesides, químico espe- } \\
\text { cialista en esta disciplina y profesor de esta universidad (Harvard). Naco-Terra Inc. } \\
\text { realizó un convenio con la Universidad de Harvard que le permitió utilizar hasta } \\
\text { cincuenta de las patentes desarrolladas dentro de la universidad. Las licencias cubren } \\
\text { auto ensamblaje molecular, litografía blanda y otros avances a escala manométrica. } \\
\text { Nano Terra está influyendo activamente en la propiedad intelectual y conocimientos } \\
\text { científicos a través de co-desarrollos, acuerdos con grandes empresas y con el gobierno } \\
\text { de los Estados Unidos. Otro ejemplo de innovación colaborativa de esta universidad } \\
\text { y que ha llegado a convertirse en una empresa con reconocimiento en el mercado es } \\
\text { el laboratorio Biogen, conocido a nivel mundial, desarrollado hace treinta años por el } \\
\text { profesor Walter Gilbert y otros colegas suyos. Esta empresa ha llegado a convertirse en } \\
\text { una de las más importantes en Biotecnología de la región de Boston, con más de } 1.500 \\
\text { empleados (Harvard, 2009). }\end{array}$ \\
\hline
\end{tabular}

Fuente: Elaboración propia. 
Tabla 4. Innovaciones colaborativas de las principales universidades de Latinoamérica

\begin{tabular}{|c|c|}
\hline Universidad & Casos exitosos de innovaciones colaborativas \\
\hline $\begin{array}{l}\text { Universidad } \\
\text { de São Paulo } \\
\text { - USP }\end{array}$ & $\begin{array}{l}\text { Cell Protect es una empresa que provee tecnología útil e innovadora en el tratamiento de } \\
\text { dolencias crónico-degenerativas humanas como la Diabetes Mellitus. Cell Protect ha de- } \\
\text { sarrollado biomateriales que realizan el micro-encapsulamiento de células pancreáticas } \\
\text { para el trasplante en pacientes diabéticos y aportan factores cito-protectores que aumen- } \\
\text { tan la oxigenación de las células encapsuladas, mejorando la funcionalidad de estas (Cell } \\
\text { Protect, 2011). Esta empresa es una start up creada en la incubadora de empresas de } \\
\text { base tecnológica CIETEC de la UsP. }\end{array}$ \\
\hline $\begin{array}{l}\text { Universidad } \\
\text { Nacional } \\
\text { Autónoma } \\
\text { de México - } \\
\text { UNAM }\end{array}$ & $\begin{array}{l}\text { Boehringer Ingelheim. Este caso de investigación colaborativa entre la Universidad } \\
\text { Nacional Autónoma de México (UNAM), a través de su instituto de Biotecnología, y la } \\
\text { empresa farmacéutica Boehringer Ingelheim, se realizó para el desarrollo de una va- } \\
\text { cuna recombinante contra la influenza aviar H5N1, que resultó de alta patogenicidad e } \\
\text { inmuniza a las aves contra esta enfermedad (Journalmex, 2011). El objetivo principal de } \\
\text { la división de extensión universitaria de la UNAM es consolidar el vínculo universidad- } \\
\text { sociedad, satisfaciendo la demanda de actualización de recursos humanos y de inserción } \\
\text { de tecnología en el sector productivo y social (Universidad Nacional Autónoma de } \\
\text { México -UNAM, 2011). }\end{array}$ \\
\hline
\end{tabular}

Fuente: Elaboración propia.

es aún un reto para las universidades de la región.

En Colombia las universidades líderes (ver Tabla 5) reflejan diferentes estrategias y mecanismos para interactuar con el entorno, especialmente a partir de las actividades de extensión o proyección social; siendo menos visibles mecanismos como los parques tecnológicos y las actividades consolidadas de emprendimiento universitario. Cabe destacar que la Universidad de Antioquia es una de las primeras universidades colombianas en desarrollar spin offs (Ruiz, 2009).

En Colombia se requiere revisar y mejorar la normativa institucional de las universidades respecto al manejo de la propiedad intelectual. Casos como la creación de la spin off Conoser en la Universidad de Antioquia, dejan como conclusión la necesidad de avanzar en estos temas para poder interactuar con el entorno de manera efectiva (Vivas, 2012).

\subsection{Características generales de la innova- ción colaborativa por región}

Con base en los resultados encontrados y estudios previos referentes al tema (Pineda et al., 2011), se infiere que las condiciones políticas, económicas, sociales, culturales y tecnológicas facilitan o inhiben las actividades colaborativas entre universidades y su entorno competitivo; razón por la cual en las regiones de Norteamérica, Europa y Asia, estas condiciones coadyuvan en el desarrollo de conocimiento, desarrollo tecnológico e innovación. En contraste, en Latinoamérica los casos de innovación señalados corresponden a casos excepcionales y por ello, exitosos, dentro de las actividades de extensión de las universidades. En este sentido, las universidades latinoamericanas están llamadas a tener claro su origen y condiciones favorables y a involucrar instituciones que actúen sinérgicamente para ofrecer oportunidades de innovación en el desarrollo económico y social de la región.

En Norteamérica, especialmente en Boston, existe un apalancamiento entre universidades, empresas y Estado, que les ha permitido trabajar conjuntamente en dar respuesta a las necesidades sociales y económicas de la región. Asimismo, las facilidades de acceso a financiación y la experiencia en el manejo de propiedad 
Tabla 5. Innovaciones colaborativas de las principales universidades de Colombia

\begin{tabular}{|c|c|}
\hline Universidad & Casos exitosos de innovaciones colaborativas \\
\hline $\begin{array}{l}\text { Universidad } \\
\text { Nacional de } \\
\text { Colombia - } \\
\text { UN (2011) }\end{array}$ & $\begin{array}{l}\text { Ecopetrol. Este es un caso de investigación colaborativa entre la Escuela de Física de la } \\
\text { Facultad de Ciencias de la un Sede Medellín y la reconocida empresa Ecopetrol, que per- } \\
\text { mitió el desarrollo de un robot para la inspección de tuberías, mediante la utilización de } \\
\text { fibra óptica. Este robot tiene la capacidad de transmitir imágenes en tiempo real para los } \\
\text { sistemas de monitoreo y control (Agencia de noticias un, 2009). Igualmente, la Universi- } \\
\text { dad ha desarrollado cuatro megaproyectos con los cuales busca integrarse con el entorno, } \\
\text { como la alianza con ParqueSoft, la Alianza Universidad Empresa Estado y el Proyecto de } \\
\text { Innovación, Gestión y Transferencia del Conocimiento. A partir de estas alianzas se han } \\
\text { generado aportes significativos al entorno. }\end{array}$ \\
\hline $\begin{array}{l}\text { Universidad } \\
\text { de Antio- } \\
\text { quia - UDEA }\end{array}$ & $\begin{array}{l}\text { Conocimiento y servicios de ingeniería s.A.s. "Conoser" es una consultora especializada } \\
\text { en servicios de manejo eficiente de la energía; cuenta con dos líneas de productos, los } \\
\text { Paquetes Tecnológicos (hechos según las necesidades de clientes industriales) y los Ser- } \\
\text { vicios (dirigidos a pymes, empresas de construcción, comerciales y otras, que necesitan } \\
\text { protección eléctrica en sus instalaciones o mejoras en el manejo de la energía). Conoser } \\
\text { está ubicada en el Parque del Emprendimiento (Ministerio de Educación Nacional, 2011). } \\
\text { Esta es la primera compañía tipo spin off universitaria creada en Colombia, derivada de } \\
\text { resultados de investigación de la Facultad de Ingeniería de la Universidad de Antioquia y } \\
\text { BIorNNoco, y financiada con recursos del Fondo de Capital de Riesgo "Capital Medellín" } \\
\text { (Universidad de Antioquia-UDEA, 2012). Según Vivas (2012) como parte del proceso de } \\
\text { creación de ésta spin off, la UDEA tuvo que hacer ajustes a su normatividad interna refer- } \\
\text { ente a la extensión universitaria. }\end{array}$ \\
\hline
\end{tabular}

Fuente: Elaboración propia.

intelectual, presentan a estas universidades como líderes mundiales en los aspectos de innovación colaborativa.

En Europa, el origen y filosofía de las universidades ha sido de tipo tradicional y con intereses más académicos y disciplinarios; lo cual refleja su menor interés en el establecimiento de vinculaciones. No obstante, las universidades inglesas han respondido dinámicamente a los cambios en las demandas de la sociedad.

El caso asiático es de destacar por la rápida adaptación de las universidades en las últimas décadas a los requerimientos del entorno, a pesar de las limitaciones en financiación, credibilidad y burocracia que aún presentan. Esto significa que estas universidades han sabido superar aspectos culturales como el idioma y tradiciones locales, adaptándose a los requerimientos de la economía global. Asia es una región líder en innovación tecnológica y son en principio las empresas las que han demandado investigación para sus innovaciones.

En América Latina las limitaciones se encuentran principalmente en aspectos culturales, caracterizados por las discusiones en torno al rol que deben tener las universidades frente a la participación en actividades colaborativas direccionadas al mercado. También, la inexperiencia en el manejo de la propiedad intelectual, los aspectos burocráticos de los Estados, los escasos recursos tecnológicos y la poca demanda de estos, ha restringido el involucramiento de las universidades, empresas y otras organizaciones en innovaciones colaborativas. Sin embargo, cabe destacar el caso brasileño, que actualmente se establece como uno de los más fuertes en toda la región.

En resumen, en todos estos casos se evidencia el propósito de innovar o realizar desarrollos tecnológicos a través de la interacción, como producto de la confianza 
y capacidad de los actores, para transferir sus distintos conocimientos e interactuar con un mismo propósito.

\section{Conclusiones}

Teniendo en cuenta el objetivo de la investigación, los resultados encontrados reflejan diversas estrategias y mecanismos para la innovación colaborativa como spin off, spin out, start ups, investigaciones aplicadas conjuntas e incubadoras de empresas que generalmente corresponden con las condiciones políticas, económicas, sociales, culturales y tecnológicas de cada región y universidad.

En la actualidad, tanto las organizaciones como las universidades requieren de un alto grado de flexibilidad y capacidad de aprendizaje para relacionarse a partir de sus capacidades e intereses, como lo reflejan las experiencias estudiadas. Se evidencia en todos los casos la tendencia a intercambiar saberes entre los distintos agentes para favorecer su competitividad y contribuir al desarrollo local y regional.

Las universidades tienen el reto de crear estructuras apropiadas que permitan la interacción efectiva con otras organizaciones del entorno o actores específicos de la sociedad, así como hacer un inventario de sus capacidades de investigación, formación y extensión, que muestre qué deben mejorar frente a sus intereses y a los requerimientos del contexto. Sin embargo, esto no puede darse por separado de la generación de reglas claras para dicha interacción, como es el caso del manejo de políticas y reglamentación sobre propiedad intelectual.

Este trabajo sirve de base de reflexión, especialmente para las universidades, en cuanto a su rol en la sociedad de generación y transferencia de conocimiento que solucione problemáticas del contexto local, regional e internacional. De igual forma, es un llamado de atención para sectores empresariales que generan valor a partir del conocimiento y la tecnología, ya que son los llamados a tomar el liderazgo del establecimiento de acciones colaborativas con la academia.

Considerando las limitaciones que se encontraron en las diferentes organizaciones y contextos socio-económicos, especialmente en el caso de Colombia, se plantea la necesidad de futuras investigaciones respecto a cómo vincular la universidad al entorno de una manera efectiva, teniendo en cuenta que el tejido productivo colombiano está constituido por MIPYMES y pertenece en su mayoría al sector tradicional de la economía, lo cual constituye una barrera y uno de los principales desafíos para la gestación de innovaciones tecnológicas; y de otra parte, especialmente en las universidades públicas, existe todavía una concepción negativa frente a la venta de servicios y conocimiento.

\section{Referencias bibliográficas}

Agencia de noticias UN. (2009). UN diseña robot para Ecopetrol. Recuperado el 12 de octubre de 2011, de http://www.agenciadenoticias.unal.edu.co/nc/videos/article/ un-disena-robot-para-ecopetrol/index. html

Baba, Y., Yarime, M. \& Shichijo, N. (2010). Sources of Success in Advanced Materials Innovation: The Role of "Core Researchers" In University- Industry Collaboration in Japan. International Journal of Innovation Management, 14(2), 201-219. Recuperado el 27 de octubre de 2011, de http://www. zzz.rcast.u-tokyo.ac.jp/IJIMo90521final-1.pdf

Casallas, C., Plata, P. \& Pineda, K. (2011). Parques Tecnológicos como Mecanismo de Integración entre Universidades, Empresas y el Estado: retos para Colombia. En Memorias VI Congreso Internacional 
de la Red de Investigación y Docencia en Innovación Tecnológica- RIDIT, Manizales, Colombia.

Cell Protect. (2011). Biotechnology. Disponible en http://www.cellprotect.com.br/ tec-produtos.aspx

CsIc. (2011). Ranking Web de Universidades del Mundo. Recuperado el 29 de junio de 2011, de http://www.webometrics.info/es

De Paula, E. \& Silveira, G. (2009). ¿E Agora, o que Fazer com Essa Tecnologia? Um Estudo Multicaso sobre as Possibilidades de Transferência de Tecnologia na USP-RP. Revista de Administração Contemporânea, 13(3), 410-429.

Enecsys. (2011). Micro Inverters. Recuperado el 12 de octubre de 2011, de http://www. enecsys.com/

Etzkowitz, H. \& Leydesdorff, L. (2000). The Dynamics of Innovation: from National Systems and Mode 2 to a Triple Helix of University-Industry-government relations. Research Policy, 29(2), 109-123.

Harvard. (2009). Investing in Innovation. Harvard University's impact on the Economy of the Boston Area. Harvard Summary, January, 1-18.

Hershberg, E., Nabeshima, K. \& Yusuf, S. (2007). Opening the Ivory Tower to Business: University-Industry Linkages and the Development of Knowledge-Intensive Clusters in Asian Cities. World Development, 35(6), 931-940.

Hughes, A. (2006) University-Industry Linkages and UK Science and Innovation Policy. University of Cambridge (Working Paper No. 326).

Intel-NTU Connected Context Computing Center. (2011). Disponible en http://ccc. ntu.edu.tw/index.php/en/

Journalmex. (2011). Se crea en la UNAM, con empresa farmacéutica, vacuna recombinante contra influenza aviar. Recuperado el 29 de julio de 2011, de http:// journalmex.wordpress.com/2011/05/08/ se-crea-en-la-unam-con-empresafarmaceutica-vacuna-recombinantecontra-influenza-aviar/

Massachusetts Institute of Technology - MIT. (2011). MIT Efficiency Forward Program Powered BY MIT and NSTAR. Recuperado el 29 de julio de 2011, de http://web.mit. edu/mitei/campus/index.html

Ministerio de Educación Nacional. (2011). Antioquia fortalece su potencial de desarrollo empresarial y científico, con la creación de la primera SPIN OFF universitaria del país. Centro Virtual de Noticias de la Educación. Recuperado el 10 de diciembre de 2010, de http://www.mineducacion.gov. $\mathrm{co} / \mathrm{cvn} / 1665 / \mathrm{w} 3$-article-258314.html

Morales, M., Mira, G. \& Arias, M. (2010). Enfoques y retos de la función de extensión universitaria como mecanismos de integración: Universidad, Empresa, Estado. Documento no publicado presentado en II Congreso Internacional de Gestión Tecnológica e Innovación, Bogotá, Colombia. Natural Taiwan University-NTU. (2011). Intel and NTU jointly established research center to explore machine to machine $\left(M_{2} M\right)$ communication technology. Highlights. Recuperado el 12 de octubre de 2011, de http://www.ntu.edu.tw/engv4/ highlights/2011/he110223_1.html

ocde \& eurostat. (2005). Manual de Oslo. Guía para la recogida e interpretación de datos sobre innovación ( $3^{\mathrm{a}}$ ed.). Madrid: Grupo Tragsa.

Oxitec. (2011). Recuperado el 27 de octubre de 2011, de http://www.oxitec.com/

Pertuzé, J., Calder, E., Greitzer, E. \& Lucas, W. (2010). Best Practices for IndustryUniversity Collaboration. MIT Sloan Management Review, 51(4), 83-90.

Pineda, K., Morales, M. \& Ortiz, C. (2011). Modelos y mecanismos de interacción universidad-empresa-Estado: retos para 
las universidades colombianas. Equidad \& Desarrollo, 15, 41-67.

Ruiz, O. (2009). El camino hacia las spin off en Medellín, Colombia. Experiencias vividas desde la Universidad de Antioquia. Recuperado el 14 de abril de 2011, de http://www.uv.es/motiva2/Ponencias\%20 Motiva2009/docs/107.pdf

Sábato, J. \& Botana, N. (1986). La ciencia y la tecnología en el desarrollo futuro en América Latina. Documento no publicado presentado en The World Order Models Conference, Bellagio, Italia.

Shahid, Y. \& Nabeshima, K. (2007). How Universities Promote Economic Growth. Washington, DC: Banco Mundial.

Shane, S. (2002). Selling University Technology: Patterns from MIT. Management Science, 48(1), 122-130.

Sutz, J. (2000). The university-industrygovernment relations in Latin America. Research Policy, 29(2), 279-290.

T2Biosystems. (2011). Science and Technology. Disponible en http://www.t2biosystems. com/Site/AboutUs/tabid/57/Default.aspx

Torres, A., Dutrenit, G., Becerra, N. \& Sampedro, J. (2009, agosto). Patrones de vinculación academia-industria: factores determinantes en el caso de México. Documento no publicado presentado en $4^{\circ}$ Congreso Internacional de Sistemas de Innovación para la Competitividad, México D.F., Mexico.

Universidad de Antioquia -UDEA. (2012). Primera Spin Off de la UdeA se crea en el país. Recuperado el 2 de noviembre de 2012, de http://www.udea.edu.co/ portal/page/portal/BibliotecaPortal/ DetalleNoticia?p_id=68618804\&p_ siteid $=37$

Universidad Nacional Autónoma de México -UNAM. (2011). La ciencia en la UNAM 2007. Recuperado el 19 de juiio de 2011, de http://www.cic-ctic.unam.mx/ cic/mas_cic/publicaciones/download/ lcunam2007.pdf

Universidad Nacional de Colombia - UN. (2011). Modalidades de extensión. Recuperado el 10 de mayo de 2011, de http:// www.unal.edu.co/extensionbog/paginas/ modalidades.html

University of Cambridge. (2011). The Cambridge Connection. Recuperado el 2 de marzo de 2011, de http://www.jbs.cam. ac.uk/aboutus/camconnections/index. html

University of Oxford. (2011). Oxford Spinout Equity Management (OSEM). Recuperado el 29 de jui io de 2011, de http://www. osem.ox.ac.uk/whatwedo/index.html

University of Tokyo. (2010). The University of Tokyo Annual Report 2010. Recuperado el 29 de julio de 2011, de http://www.ducr.utokyo.ac.jp/en/materials/pdf/2009annual_ report_en.pdf

Vivas, M. (2012). ¿Pueden las universidades públicas crear Spin-Off? Memoria del Encuentro de Vicerrectores de Investigación de Universidades Públicas Colombianas. Medellín, Colombia: Universidad de Antioquia. Recuperado el 20 de abril de 2012, de http://www.udea.edu.co/portal/page/ portal/BibliotecaInvestigacion/InformacionGeneral/utilidades/documentos/ Relatoria\%20Spin\%20Off.pdf 